\title{
The Best Finite-Difference Scheme for the Helmholtz Equation
}

\author{
T. Zhanlav ${ }^{1}$, V. Ulziibayar ${ }^{2}$ \\ ${ }^{1}$ School of Mathematics and Computer Science, National University of Mongolia, Ulaanbaatar, Mongolia \\ ${ }^{2}$ School of Mathematics, Mongolian University of Science and Technology, Ulaanbaatar, Mongolia \\ Email: v_ulzii@yahoo.com
}

Received June 6, 2012; revised August 8, 2012; accepted August 12, 2012

\begin{abstract}
The best finite-difference scheme for the Helmholtz equation is suggested. A method of solving obtained finite-difference scheme is developed. The efficiency and accuracy of method were tested on several examples.
\end{abstract}

Keywords: The Best Finite-Difference Scheme for the Helmholtz and Laplace's Equations

\section{Introduction}

The finite-difference method is a standard numerical method for solving boundary value problems. Recently, considerable attention has been attracted to construct a best (or exact) difference approximation for some ordinary and partial differential equations [1-3]. In this paper a best finite-difference method is developed for Helmholtz equation with general boundary conditions on the rectangular domain in $R^{2}$. The method proposed here comes out from [4] and is based on separation of variables method and expansion of one-dimensional threepoint difference operators for sufficiently smooth solution. The paper is organized as follows. The statement of problem and the separation of variables method are considered in Section 2. A detailed description of the best difference approximation to the Helmholtz equation in rectangular domain is given in Section 3.

Section 4 is devoted to derive the best approximation for the given third kind boundary conditions. The method of solution for the obtained difference equations is considered in Section 5 and numerical examples are given last Section 6.

\section{Statement of Problem}

Let $\Omega=(a, b) \times(c, b)$ be an open rectangular domain in Euclidean $R^{2}$ space with boundary given by $\partial \Omega$. The aim is to determine a function $u(x, y)$, satisfying equation

$$
\Delta u+C u=0,(x, y) \in \Omega
$$

with boundary condition

$$
\alpha u+\beta \frac{\partial u}{\partial n}=\psi(x, y),(x, y) \in \partial \Omega,
$$

where $C$ in (2.1) is a given number and $\frac{\partial}{\partial n}$ is the outward normal on $\partial \Omega$.

It is well known that the stabilized oscillation problems and diffusing processes in gas lead to the so called Helmholtz Equation (2.1) with a positive coefficient $C=\lambda^{2}$. The diffusing process in the moving field leads to the Equation (2.1) with negative coefficient $C=-\lambda^{2}$. If $C=0$ the Equation (2.1) leads to Laplace's ones. Obviously, the properties of the solution of Equation (2.1) depend essentially upon the sign of the coefficient $C$ in (2.1). We will assume that the problem (2.1), (2.2) has an unique and sufficiently smooth solution.

By virtue of variables method looking for the solution $u(x, y)$ of Equation (2.1), (2.2) in the form

$$
u(x, y)=U_{1}(x) U_{2}(y)
$$

we arrive at equation

$$
\frac{U_{1}^{\prime \prime}(x)}{U_{1}(x)}+\frac{U_{2}^{\prime \prime}(y)}{U_{2}(y)}=-C,
$$

which is splitted into two independing equations

$$
U_{1}^{\prime \prime}(y)=\omega U_{1}(x)
$$

and

$$
U_{2}^{\prime \prime}(y)=\beta U_{2}(y), \beta=-C-\omega,
$$

where the unknown separation constant $\omega$ is to be found.

By virtue of (2.3) the boundary condition (2.2) is splitted info ones for $U_{1}(x)$ and $U_{2}(y)$

$$
\begin{aligned}
& \alpha_{1} U_{1}(a)-\beta_{1} U_{1}^{\prime}(a)=x_{10}, \\
& \alpha_{2} U_{1}(b)+\beta_{2} U_{1}^{\prime}(b)=x_{2 N}
\end{aligned}
$$


and

$$
\begin{aligned}
& \alpha_{3} U_{2}(c)-\beta_{3} U_{2}^{\prime}(c)=x_{30}, \\
& \alpha_{4} U_{2}(d)+\beta_{4} U_{2}^{\prime}(d)=x_{4 N}
\end{aligned}
$$

The solution of boundary value problem $(2.4 a),(2.5)$ is founded in a closed from

$$
U_{1}(x)=\frac{x_{10} F_{1}(\omega)+x_{2 N} F_{2}(\omega)}{F_{3}(\omega)+\sqrt{\omega} F_{4}(\omega)},
$$

where

$$
\begin{gathered}
F_{1}(\omega)=\alpha_{2} \operatorname{sh}(\sqrt{\omega}(b-x))+\beta_{2} \sqrt{\omega} \operatorname{ch}(\sqrt{\omega}(b-x)), \\
F_{2}(\omega)=\alpha_{1} \operatorname{sh}(\sqrt{\omega}(x-a))+\beta_{1} \sqrt{\omega} \operatorname{ch}(\sqrt{\omega}(x-a)), \\
\omega>0
\end{gathered}
$$

and

$$
\begin{gathered}
F_{3}(\omega)=\left(\alpha_{1} \alpha_{2}+\beta_{1} \beta_{2}\right) \operatorname{sh}(\sqrt{\omega}(b-a)), \\
F_{4}(\omega)=\left(\alpha_{1} \beta_{2}+\alpha_{2} \beta_{1}\right) \operatorname{ch}(\sqrt{\omega}(b-a)) . \\
\omega>0
\end{gathered}
$$

When $\omega<0$ the functions sh and $c h$ in (2.7) are to be replaced by $\sin$ and $\cos$ respectively and $\omega$ replaced by $-\omega$. Analogously, we can find the solutions of boundary value problem (2.4b) and (2.6) in closed form. Then from (2.3) and (2.7) clear, that the problem consists in determining the separation constant $\omega$.

\section{Construction of the Best Finite-Difference Equations}

For the numerical solution of problem (2.1), (2.2) is introduced the uniform rectangular grid $\Omega_{h}$ :

$$
\begin{aligned}
\Omega_{h}= & \left\{\left(x_{i}, y_{j}\right) \mid x_{i}=x_{0}+i h_{1} ; y_{j}=y_{0}+j h_{2} ;\right. \\
& i=0,1 \cdots, N ; j=0,1 \cdots, M\}
\end{aligned}
$$

where $h_{1}=\frac{(b-a)}{N}$ and $h_{2}=\frac{(d-c)}{M}$ are the mesh sizes in the $x$ and $y$ directions respectively. Usually, the Equation (2.1) is approximated by the five-point difference equation

$$
\begin{gathered}
\frac{y_{i+1, j}-2 y_{i, j}+y_{i-1, i}}{h_{1}^{2}}+\frac{y_{i, j+1}-2 y_{i, j}+y_{i, j-1}}{h_{2}^{2}}+C y_{i, j}=0, \\
i=1, \cdots, N-1, \quad j=1, \cdots, M-1 .
\end{gathered}
$$

The local discretization error of the Equation (3.1) is of $O\left(h_{1}^{2}+h_{2}^{2}\right)$ order. Now we describe how to derive the best difference scheme for Equation (2.1). To this end, we consider expression

$$
\left(\Lambda_{1}+\Lambda_{2}\right) u_{i j}=\frac{u_{i+1, j}-2 u_{i, j}+u_{i-1, i}}{h_{1}^{2}}+\frac{u_{i, j+1}-2 u_{i, j}+u_{i, j-1}}{h_{2}^{2}}
$$

where $u_{i, j}=u\left(x_{i}, y_{j}\right)$. If we denote by $U_{1 i}, U_{2 i}$ the values of $U_{1 i}, U_{2 j}$ the values of $U_{1}\left(x_{i}\right)$ and $U_{2}\left(y_{j}\right)$ respectively, the using (2.3) the Equation (3.2) may be written as

$$
\left(\Lambda_{1}+\Lambda_{2}\right) u_{i j}=U_{2 j} \Lambda_{1} U_{1 i}+U_{1 i} \Lambda_{2} U_{2 j} .
$$

Due to smoothness assumption of solution $u(x, y)$, as well as, functions $U_{1}(x)$ and $U_{2}(y)$, the Taylor series expansion yields

$$
\begin{aligned}
& \Lambda_{1} U_{1 i}=U_{1}^{\prime \prime}\left(x_{i}\right)+2 \sum_{k=1}^{\infty} \frac{h_{1}^{2 k} U_{1}^{2 k+2}\left(x_{i}\right)}{(2 k+2) !}, \\
& \Lambda_{2} U_{2 j}=U_{2}^{\prime \prime}\left(y_{j}\right)+2 \sum_{k=1}^{\infty} \frac{h_{2}^{2 k} U_{2}^{2 k+2}\left(y_{j}\right)}{(2 k+2) !}
\end{aligned}
$$

Because of (2.4) we have

$$
\begin{gathered}
U_{1}^{(2 k)}=\omega^{k} U_{1}, U_{2}^{(2 k)}=\beta^{k} U_{2}, \\
k=1,2, \cdots
\end{gathered}
$$

Taking into account (3.4), (3.5) in (3.3) it follows that

$$
\begin{gathered}
\left\{\Lambda_{1}+\Lambda_{2}+\left(C-2 \sum_{k=1}^{\infty} \frac{h_{1}^{2 k} \omega^{k+1}+h_{2}^{2 k} \beta^{k+1}}{(2 k+2) !}\right) E\right\} u_{i j}=0, \\
i=1,2, \cdots, N-1 ; j=1,2, \cdots, M-1,
\end{gathered}
$$

where $E$ is unit operator. The difference Equation (3.6) contains unknown nonzero parameter $\omega$ and therefore it may be considered as a nonlinear equation with respect to the parameter $\omega$ and $u_{i j}$. The series in (3.6) may be expressed through analytical functions depending on the sign of quantities $\omega$ and $\beta$ and thereby the Equation (3.6) can be rewritten as

$$
\left(\Lambda_{1}+\Lambda_{2}-2 D(\omega) E\right) u=0,(x, y) \in \Omega_{h} .
$$

There are three cases:

1) Let $C=\lambda^{2}>0$. Then it is easy to show that

$$
D= \begin{cases}\frac{\cos \left(\sqrt{-\omega} h_{1}\right)-1}{h_{1}^{2}}+\frac{\operatorname{ch}\left(\sqrt{\beta} h_{2}-1\right)}{h_{2}^{2}} & \text { if } \omega \in\left(-\infty,-\lambda^{2}\right), \\ \frac{\cos \left(\sqrt{-\omega} h_{1}\right)-1}{h_{1}^{2}}+\frac{\cos \left(\sqrt{-\beta} h_{2}-1\right)}{h_{2}^{2}} & \text { if } \omega \in\left(-\lambda^{2}, 0\right), \\ \frac{\operatorname{ch}\left(\sqrt{\omega} h_{1}\right)-1}{h_{1}^{2}}+\frac{\cos \left(\sqrt{-\beta} h_{2}-1\right)}{h_{2}^{2}} & \text { if } \omega \in(0,+\infty),\end{cases}
$$

2) Let $C=0$. In this case $D$ is given by 


$$
D= \begin{cases}\frac{\operatorname{ch}\left(\sqrt{\omega} h_{1}\right)-1}{h_{1}^{2}}+\frac{\cos \left(\sqrt{\omega} h_{2}\right)-1}{h_{2}^{2}} & \text { if } \omega>0 \\ \frac{\cos \left(\sqrt{-\omega} h_{1}\right)-1}{h_{1}^{2}}+\frac{\operatorname{ch}\left(\sqrt{-\omega} h_{2}\right)-1}{h_{2}^{2}} & \text { if } \omega<0\end{cases}
$$

3) Let $C=-\lambda^{2}<0$. In this case $D$ is given by

$$
D= \begin{cases}\frac{\cos \left(\sqrt{-\omega} h_{1}\right)-1}{h_{1}^{2}}+\frac{\operatorname{ch}\left(\sqrt{\beta} h_{2}\right)-1}{h_{2}^{2}} & \text { if } \omega<0 \\ \frac{\operatorname{ch}\left(\sqrt{-\omega} h_{1}\right)-1}{h_{1}^{2}}+\frac{\operatorname{ch}\left(\sqrt{\beta} h_{2}\right)-1}{h_{2}^{2}} & \text { if } \omega \in\left(0, \lambda^{2}\right) \\ \frac{\operatorname{ch}\left(\sqrt{\omega} h_{1}\right)-1}{h_{1}^{2}}+\frac{\operatorname{ch}\left(\sqrt{-\beta} h_{2}\right)-1}{h_{2}^{2}} & \text { if } \omega>\lambda^{2}\end{cases}
$$

Thus we obtain the best (or exact) five-point difference Equation (3.7) for the Equation (2.1) (see, for example, Mickens [2] and Agarwal [1]). The function $D(\omega)$ in (3.7) can be presented as a sum of two ones, i.e.,

$$
D(\omega)=D_{1}(\omega)+D_{1}(\omega),
$$

where $D_{1}(\omega)$ and $D_{2}(\omega)$ correspond to the first and second terms in (3.8), (3.9) and (3.10) respectively.

\section{The Best Finite-Difference Boundary Condition}

Now we will derive a best difference boundary condition for (2.5), (2.6). Using (2.4) in the Taylor series expansion

$$
U_{11}=U_{10}+h_{1} U_{10}^{\prime}+\frac{h_{1}^{2}}{2 !} U_{10}^{\prime \prime}+\frac{h_{1}^{3}}{3 !} U_{10}^{\prime \prime \prime}+\cdots
$$

we obtain

$$
U_{11}= \begin{cases}\operatorname{ch}\left(\sqrt{\omega} h_{1}\right) U_{10}+\frac{\operatorname{sh}\left(\sqrt{\omega} h_{1}\right)}{\sqrt{\omega}} U_{10}^{\prime}, & \omega>0 \\ \cos \left(\sqrt{-\omega} h_{1}\right) U_{10}+\frac{\sin \left(\sqrt{-\omega} h_{1}\right)}{\sqrt{-\omega}} U_{10}^{\prime}, \quad \omega<0\end{cases}
$$

If $\beta_{1}=0$ in (2.5), then we have

$$
U_{10}=\frac{x_{10}}{\alpha_{1}}
$$

If $\beta_{1} \neq 0$, then finding $U_{10}^{\prime}$ from (2.5) and substituting it in (4.1) we get

$$
U_{11}=\theta_{1}(\omega) U_{10}+\theta_{2}(\omega),
$$

where $\theta_{1}(\omega)$ and $\theta_{2}(\omega)$ are given by

$$
\theta_{1}(\omega)= \begin{cases}\operatorname{ch}\left(\sqrt{\omega} h_{1}\right)+\frac{\alpha_{1}}{\beta_{1}} \frac{\operatorname{sh}\left(\sqrt{\omega} h_{1}\right)}{\sqrt{\omega}}, & \omega>0 \\ \cos \left(\sqrt{-\omega} h_{1}\right)+\frac{\alpha_{1}}{\beta_{1}} \frac{\sin \left(\sqrt{-\omega} h_{1}\right)}{\sqrt{-\omega}}, & \omega<0\end{cases}
$$

and

$$
\theta_{2}(\omega)= \begin{cases}-\frac{x_{10}}{\beta_{1}} \frac{\operatorname{sh}\left(\sqrt{\omega} h_{1}\right)}{\sqrt{\omega}}, & \omega>0 \\ -\frac{x_{10}}{\beta_{1}} \frac{\sin \left(\sqrt{-\omega} h_{1}\right)}{\sqrt{-\omega}}, & \omega<0\end{cases}
$$

Analogously, it is easy to verify that the exact difference boundary condition for $U_{1}(x)$ at point $x=b$ is given by

$$
\begin{gathered}
U_{1 N}=\frac{X_{2 N}}{\alpha_{2}} \text { when } \beta_{2}=0, \\
U_{1 N-1}=\theta_{3}(\omega) U_{1 N-1}+\theta_{4}(\omega) \text {, when } \beta_{2} \neq 0,
\end{gathered}
$$

where $\theta_{3}(\omega)$ and $\theta_{4}(\omega)$ are given by

$$
\theta_{3}(\omega)= \begin{cases}\operatorname{ch}\left(\sqrt{\omega} h_{1}\right)+\frac{\alpha_{2}}{\beta_{2}} \frac{\operatorname{sh}\left(\sqrt{\omega} h_{1}\right)}{\sqrt{\omega}} & \omega>0 \\ \cos \left(\sqrt{-\omega} h_{1}\right)+\frac{\alpha_{2}}{\beta_{2}} \frac{\sin \left(\sqrt{-\omega} h_{1}\right)}{\sqrt{-\omega}} & \omega<0\end{cases}
$$

and

$$
\theta_{4}(\omega)= \begin{cases}-\frac{x_{2 N}}{\beta_{2}} \frac{\operatorname{sh}\left(\sqrt{\omega} h_{1}\right)}{\sqrt{\omega}} & \omega>0 \\ -\frac{x_{2 N}}{\beta_{2}} \frac{\sin \left(\sqrt{-\omega} h_{1}\right)}{\sqrt{-\omega}} & \omega<0\end{cases}
$$

In the same way, as before, one can construct the best difference boundary conditions for $U_{2}(y)$. We omit the evaluation and present only the final results:

$$
\begin{gathered}
U_{20}=\frac{X_{30}}{\alpha_{3}} \text {, when } \beta_{3}=0, \\
U_{21} \bar{\theta}_{1}(\omega) U_{20}+\bar{\theta}_{2}(\omega) \text {, when } \beta_{3} \neq 0,
\end{gathered}
$$

and

$$
\begin{gathered}
U_{2 M}=\frac{X_{4 M}}{\alpha_{4}} \text {, when } \beta_{4}=0 \\
U_{2, M-1} \bar{\theta}_{3}(\omega) U_{2, M}+\bar{\theta}_{4}(\omega) \text {, when } \beta_{4} \neq 0
\end{gathered}
$$

where $\bar{\theta}_{i}(\omega)$ are defined by 


$$
\begin{gathered}
\bar{\theta}_{1}(\omega)= \begin{cases}\cos \left(\sqrt{\beta} h_{2}\right)+\frac{\alpha_{3}}{\beta_{3}} \frac{\sin \left(\sqrt{\beta} h_{2}\right)}{\sqrt{\beta}}, \quad \beta>0 \\
\operatorname{ch}\left(\sqrt{-\beta} h_{1}\right)+\frac{\alpha_{3}}{\beta_{3}} \frac{\operatorname{sh}\left(\sqrt{-\beta} h_{2}\right)}{\sqrt{-\beta}}, \quad \beta<0\end{cases} \\
\bar{\theta}_{2}(\omega)= \begin{cases}-\frac{x_{30}}{\beta_{3}} \frac{\sin \left(\sqrt{\beta} h_{2}\right)}{\sqrt{\beta}}, & \beta>0 \\
-\frac{x_{30}}{\beta_{3}} \frac{\operatorname{sh}\left(\sqrt{-\beta} h_{2}\right)}{\sqrt{-\beta}}, & \beta<0\end{cases}
\end{gathered}
$$

and

$$
\begin{gathered}
\bar{\theta}_{3}(\omega)= \begin{cases}\cos \left(\sqrt{\beta} h_{2}\right)+\frac{\alpha_{4}}{\beta_{4}} \frac{\sin \left(\sqrt{\beta} h_{2}\right)}{\sqrt{\beta}}, \quad \beta>0 \\
\operatorname{ch}\left(\sqrt{-\beta} h_{2}\right)+\frac{\alpha_{4}}{\beta_{4}} \frac{\operatorname{sh}\left(\sqrt{-\beta} h_{2}\right)}{\sqrt{-\beta}}, \quad \beta<0\end{cases} \\
\bar{\theta}_{4}(\omega)= \begin{cases}-\frac{x_{4 M}}{\beta_{4}} \frac{\sin \left(\sqrt{\beta} h_{2}\right)}{\sqrt{\beta}}, & \beta>0 \\
-\frac{x_{4 M}}{\beta_{4}} \frac{\operatorname{sh}\left(\sqrt{-\beta} h_{2}\right)}{\sqrt{-\beta}}, & \beta<0\end{cases}
\end{gathered}
$$

\section{Method for Solution of Finite-Difference Equations}

In this section we consider a method for solving the finite-difference Equations (3.7). For this purpose we rewrite it in the from

$$
\begin{gathered}
U_{2 j}\left(\Lambda_{1}-2 D_{1}(\omega) E\right) U_{1 i}+U_{1 i}\left(\Lambda_{2}-2 D_{2}(\omega) E\right) U_{2 j}=0, \\
i=1, \cdots, N-1, \quad j=1, \cdots, M-1,
\end{gathered}
$$

in which we have used (3.11) From this it is clear, that Equation (5.1) will be fulfilled if we choose $U_{1 i}$ and $U_{2 j}$ such that

$$
\begin{aligned}
& \left(\Lambda_{1}-2 D_{1}(\omega) E\right) U_{1 i}=0, i=1, \cdots, N-1, \\
& \left(\Lambda_{2}-2 D_{2}(\omega) E\right) U_{2 i}=0, j=1, \cdots, M-1
\end{aligned}
$$

The last weakly coupled system of Equation (5.2) is splitted into two equations with corresponding boundary conditions. First, we consider the Equation (5.2a) subject to boundary conditions (4.2) and (4.4).

According to (2.1), (2.2) and (2.3) the function $U_{1}(x)$ will be defermined within an arbitrary multiplicative constant. Therefore the three-point finite-difference Equation (5.2a) can be solved by shooting method starting with $U_{10}^{0}, U_{11}^{0}$ and $\omega_{0}$ which are required to be known. Thanks to (4.2) it is possible to find $U_{10}$ or $U_{11}$ depending on the $\beta_{1}$. For example, if $\beta_{1}=0$ then $U_{10}$ is determined by (4.2a) and $U_{11}$ and $\omega$ to be chosen arbitrary. Otherwise, $U_{11}$ is determined by (4.2b) and $U_{10}$ and $\omega$ to be chosen arbitrary.

Note, that when $C \neq 0$ one of the boundary conditions (2.5), (2.6) is assumed to be homogeneous. For Laplace's equation we always can leads to equation with homogeneous boundary conditions by change of variables. The exact value of parameter $\omega$ must satisfy

$$
\Phi(\omega)=0
$$

where $\Phi(\omega)$, for examples, when $X_{10}=0$ defined by

$$
\Phi(\omega)= \begin{cases}U_{1 N}-\frac{1}{\alpha_{2}} X_{2 N}, & \text { when } \beta_{2}>0 \\ U_{1, N-1}-\theta_{3}(\omega) U_{1 N}-\theta_{4}(\omega), & \text { when } \beta_{2} \neq 0\end{cases}
$$

The nonlinear Equation (5.3) can be solved by Newton's method:

$$
\omega_{k+1}=\omega_{k}-\frac{\Phi\left(\omega_{k}\right)}{\Phi^{\prime}\left(\omega_{k}\right)}, k=0,1,2, \cdots
$$

The value $\Phi^{\prime}\left(\omega_{k}\right)$ in the dominator of (5.5) is found by differentiating the Equation (5.4) and (5.2a) with respect to $\omega$. The iteration process $(5.5)$ is terminated by criterion

$$
\left|\omega_{k+1}-\omega_{k}\right| \leq \mathcal{E}
$$

where $\mathcal{E}$ is a reassigned accuracy.

If the evaluation of $\Phi^{\prime}(\omega)$ causes some difficulty we can use secant method instead of Newton's ones. After finding $\omega$ the three-point difference equations (5.2b) with boundary conditions (4.6), (4.7) can be solved by elimination method.

\section{Numerical Results}

We have tested the efficiency and accuracy of finitedifference scheme (3.7) on the several examples.

Example 1.

$$
\Delta u=0,0 \leq x, y \leq 1
$$

with boundary condition

$$
\begin{gathered}
u(0, y)-u^{\prime}(0, y)=6 \pi \frac{\operatorname{sh}(6 \pi y)}{\operatorname{sh}(6 \pi)}, u(1, y)=0, \\
u(x, 0)=0, u(x, 1)=\sin (6 \pi x) .
\end{gathered}
$$

The exact solution is given by

$$
u(x, y)=\sin (6 \pi x) \cdot \frac{\operatorname{sh}(6 \pi y)}{\operatorname{sh}(6 \pi)} .
$$

In Table 1 we present the computed values of $u_{i j}=U_{1 i} U_{2 j}$ (exact values of $u_{i j}=u\left(x_{i}, y_{j}\right)$ present in 
brackets) for $N=5$ and $M=4$. In order to use secant method we need two first approximations $\omega_{0}$ and $\omega_{1}$ to $\omega$. The iteration was terminated by criterion (5.6) with $\mathcal{E}=10^{-7}$.

Example 2.

$$
\Delta u=0,0 \leq x, y \leq 1
$$

with boundary condition

$$
\begin{gathered}
u(0, y)=u(1, y)=0, \\
u(x, 0)=0, u(x, 1)=\sin (\pi x) .
\end{gathered}
$$

The exact solution is given by

$$
u(x, y)=\sin (\pi x) \cdot \frac{\operatorname{sh}(\pi y)}{\operatorname{sh}(\pi)},
$$

In Table 2 we present the computed values of $u_{i j}=U_{1 i} U_{2 j}$ (exact values of $u_{i j}=u\left(x_{i}, y_{j}\right)$ present in brackets) for $N=6$ and $M=6$. In order to use secant method we need two first approximations $\omega_{0}$ and $\omega_{1}$ to $\omega$. In this example choose $\omega_{0}=-5$ and $\omega_{1}=-6$. The exact value of $\omega$ is $\omega=-\pi^{2}$. The convergence of $\omega_{k}$ was tabulated in Table 3 . The iteration was terminated by criterion (5.6) with $\mathcal{E}=10^{-7}$.

Example 3.

$$
\Delta u+2 u=0,0 \leq x, y \leq 1
$$

with boundary condition

$$
\begin{gathered}
u(0, y)-u^{\prime}(0, y)=0, u(1, y)=\exp (1+y), \\
u(x, 0)=\exp (x), u(x, 1)=\exp (1+x) .
\end{gathered}
$$

\begin{tabular}{|c|c|c|c|c|c|c|}
\hline$u_{i, j}$ & 0 & 1 & 2 & 3 & 4 & 5 \\
\hline 4 & $\begin{array}{c}0.0000000 \\
(0.0000000)\end{array}$ & $\begin{array}{c}-0.5877853 \\
(-0.5877853)\end{array}$ & $\begin{array}{c}0.9510565 \\
(0.9510565)\end{array}$ & $\begin{array}{c}-0.9510565 \\
(-0.9510565)\end{array}$ & $\begin{array}{c}0.5877853 \\
(0.5877853)\end{array}$ & $\begin{array}{c}0.0000000 \\
(0.0000000)\end{array}$ \\
\hline 3 & $\begin{array}{c}0.0000000 \\
(0.0000000)\end{array}$ & $\begin{array}{c}-0.0052802 \\
(-0.0052802)\end{array}$ & $\begin{array}{c}0.0085436 \\
(0.0085436)\end{array}$ & $\begin{array}{c}-0.0085436 \\
(-0.0085436)\end{array}$ & $\begin{array}{c}0.0052802 \\
(0.0052802)\end{array}$ & $\begin{array}{c}0.0000000 \\
(0.0000000)\end{array}$ \\
\hline 2 & $\begin{array}{c}0.0000000 \\
(0.0000000)\end{array}$ & $\begin{array}{c}-0.0000474 \\
(-0.0000474)\end{array}$ & $\begin{array}{c}0.0000767 \\
(0.0000767)\end{array}$ & $\begin{array}{c}-0.0000767 \\
(-0.0000767)\end{array}$ & $\begin{array}{c}0.0000474 \\
(0.0000474)\end{array}$ & $\begin{array}{c}0.0000000 \\
(0.0000000)\end{array}$ \\
\hline 1 & $\begin{array}{c}0.0000000 \\
(0.0000000)\end{array}$ & $\begin{array}{c}-0.0000004 \\
(-0.0000004)\end{array}$ & $\begin{array}{c}0.0000007 \\
(0.0000007)\end{array}$ & $\begin{array}{c}-0.0000007 \\
(-0.0000007)\end{array}$ & $\begin{array}{c}0.0000004 \\
(0.0000004)\end{array}$ & $\begin{array}{c}0.0000000 \\
(0.0000000)\end{array}$ \\
\hline 0 & $\begin{array}{c}0.0000000 \\
(0.0000000)\end{array}$ & $\begin{array}{c}0.0000000 \\
(0.0000000)\end{array}$ & $\begin{array}{c}0.0000000 \\
(0.0000000)\end{array}$ & $\begin{array}{c}0.0000000 \\
(0.0000000)\end{array}$ & $\begin{array}{c}0.0000000 \\
(0.0000000)\end{array}$ & $\begin{array}{c}0.0000000 \\
(0.0000000)\end{array}$ \\
\hline
\end{tabular}

The exact solution is given by

$$
u(x, y)=\exp (x+y),
$$

\begin{tabular}{|c|c|c|c|c|c|c|c|}
\hline$u_{i, j}$ & 0 & 1 & 2 & 3 & 4 & 5 & 6 \\
\hline 6 & $\begin{array}{c}0.0000000 \\
(0.0000000)\end{array}$ & $\begin{array}{c}0.5000000 \\
(0.5000000)\end{array}$ & $\begin{array}{c}0.8660254 \\
(0.8660254)\end{array}$ & $\begin{array}{c}1.0000000 \\
(1.0000000)\end{array}$ & $\begin{array}{c}0.8660254 \\
(0.8660254)\end{array}$ & $\begin{array}{c}0.5000000 \\
(0.5000000)\end{array}$ & $\begin{array}{l}-0.0000000 \\
(0.0000000)\end{array}$ \\
\hline 5 & $\begin{array}{c}0.0000000 \\
(0.0000000)\end{array}$ & $\begin{array}{c}0.2951674 \\
(0.2951674)\end{array}$ & $\begin{array}{c}0.5112450 \\
(0.5112450)\end{array}$ & $\begin{array}{c}0.5903348 \\
(0.5903348)\end{array}$ & $\begin{array}{c}0.5112450 \\
(0.5112450)\end{array}$ & $\begin{array}{c}0.2951674 \\
(0.2951674)\end{array}$ & $\begin{array}{c}-(0.0000000) \\
(0.0000000)\end{array}$ \\
\hline 4 & $\begin{array}{c}0.0000000 \\
(0.0000000)\end{array}$ & $\begin{array}{c}0.1731224 \\
(0.1731224)\end{array}$ & $\begin{array}{c}0.2998568 \\
(0.2998568)\end{array}$ & $\begin{array}{c}0.3462448 \\
(0.3462448)\end{array}$ & $\begin{array}{c}0.2998568 \\
(0.2998568)\end{array}$ & $\begin{array}{c}0.1731224 \\
(0.1731224)\end{array}$ & $\begin{array}{l}-0.0000000 \\
(0.0000000)\end{array}$ \\
\hline 3 & $\begin{array}{c}0.0000000 \\
(0.0000000)\end{array}$ & $\begin{array}{c}0.0996342 \\
(0.0996342)\end{array}$ & $\begin{array}{c}0.1725715 \\
(0.1725715)\end{array}$ & $\begin{array}{c}0.1992684 \\
(0.1992684)\end{array}$ & $\begin{array}{c}0.1725715 \\
(0.1725715)\end{array}$ & $\begin{array}{c}0.0996342 \\
(0.0996342)\end{array}$ & $\begin{array}{l}-0.0000000 \\
(0.0000000)\end{array}$ \\
\hline 2 & $\begin{array}{c}0.0000000 \\
(0.0000000)\end{array}$ & $\begin{array}{c}0.0540911 \\
(0.0540911)\end{array}$ & $\begin{array}{c}0.0936885 \\
(0.0936885)\end{array}$ & $\begin{array}{c}0.1081821 \\
(0.1081821)\end{array}$ & $\begin{array}{c}0.0936885 \\
(0.0936885)\end{array}$ & $\begin{array}{c}0.0540911 \\
(0.0540911)\end{array}$ & $\begin{array}{l}-0.0000000 \\
(0.0000000)\end{array}$ \\
\hline 1 & $\begin{array}{c}0.0000000 \\
(0.0000000)\end{array}$ & $\begin{array}{c}0.0237192 \\
(0.0237192)\end{array}$ & $\begin{array}{c}0.0410828 \\
(0.0410828)\end{array}$ & $\begin{array}{c}0.0474384 \\
(0.0474384)\end{array}$ & $\begin{array}{c}0.0410828 \\
(0.0410828)\end{array}$ & $\begin{array}{c}0.0237192 \\
(0.0237192)\end{array}$ & $\begin{array}{l}-0.0000000 \\
(0.0000000)\end{array}$ \\
\hline 0 & $\begin{array}{c}0.0000000 \\
(0.0000000)\end{array}$ & $\begin{array}{c}0.0000000 \\
(0.0000000)\end{array}$ & $\begin{array}{c}0.0000000 \\
(0.0000000)\end{array}$ & $\begin{array}{c}0.0000000 \\
(0.0000000)\end{array}$ & $\begin{array}{c}0.0000000 \\
(0.0000000)\end{array}$ & $\begin{array}{c}0.0000000 \\
(0.0000000)\end{array}$ & $\begin{array}{c}0.0000000 \\
(0.0000000)\end{array}$ \\
\hline 0 & $\begin{array}{c}0.0000000 \\
(0.0000000)\end{array}$ & $\begin{array}{c}0.0000000 \\
(0.0000000)\end{array}$ & $\begin{array}{c}0.0000000 \\
(0.0000000)\end{array}$ & $\begin{array}{c}0.0000000 \\
(0.0000000)\end{array}$ & $\begin{array}{c}0.0000000 \\
(0.0000000)\end{array}$ & $\begin{array}{c}0.0000000 \\
(0.0000000)\end{array}$ & $\begin{array}{c}0.0000000 \\
(0.0000000)\end{array}$ \\
\hline
\end{tabular}

In Table 4 we present the computed values of $u_{i j}=U_{1 i} U_{2 j}$ (exact values of $u_{i j}=u\left(x_{i}, y_{j}\right)$ present in brackets) for $N=6$ and $M=4$. In order to use secant

Table 1. Computed values of $u_{i j}=U_{1 i} U_{2 j}$ for $N=5$ and $M=4$.

Table 2. Computed values of $u_{i j}=U_{1 i} U_{2 j}$ for $N=6$ and $M=6$. 
Table 3. The convergence of $\omega_{k}$.

\begin{tabular}{cccccc}
\hline$\omega_{2}$ & $\omega_{3}$ & $\omega_{4}$ & $\omega_{5}$ & $\omega_{6}$ & $\omega_{7}$ \\
\hline-8.4944719 & -9.4982904 & -9.8358860 & -9.8687281 & -9.8696023 & -9.86960448 \\
\hline
\end{tabular}

Table 4. Computed values of $u_{i j}=U_{1 i} U_{2 j}$ for $N=6$ and $M=4$.

\begin{tabular}{|c|c|c|c|c|c|c|c|}
\hline$u_{i, j}$ & 0 & 1 & 2 & 3 & 4 & 5 & 6 \\
\hline 4 & $\begin{array}{c}2.7182818 \\
(2.7182818)\end{array}$ & $\begin{array}{c}3.2112705 \\
(3.2112705)\end{array}$ & $\begin{array}{c}3.7936679 \\
(3.7936679)\end{array}$ & $\begin{array}{c}4.4816891 \\
(4.4816891)\end{array}$ & $\begin{array}{c}5.2944901 \\
(5.2944901)\end{array}$ & $\begin{array}{c}6.2547010 \\
(6.2547010)\end{array}$ & $\begin{array}{c}7.3890561 \\
(7.3890561)\end{array}$ \\
\hline 3 & $\begin{array}{c}2.1170000 \\
(2.1170000)\end{array}$ & $\begin{array}{c}2.5009400 \\
(2.5009400)\end{array}$ & $\begin{array}{c}2.9545115 \\
(2.9545115)\end{array}$ & $\begin{array}{c}3.4903430 \\
(3.4903430)\end{array}$ & $\begin{array}{c}4.1233530 \\
(4.1233530)\end{array}$ & $\begin{array}{c}4.8711660 \\
(4.8711660)\end{array}$ & $\begin{array}{c}5.7546027 \\
(5.7546027)\end{array}$ \\
\hline 2 & $\begin{array}{c}1.6487213 \\
(1.6487213)\end{array}$ & $\begin{array}{c}1.9477340 \\
(1.9477340)\end{array}$ & $\begin{array}{c}2.3009759 \\
(2.3009759)\end{array}$ & $\begin{array}{c}2.7182818 \\
(2.7182818)\end{array}$ & $\begin{array}{c}3.2112705 \\
(3.2112705)\end{array}$ & $\begin{array}{c}3.7936679 \\
(3.7936679)\end{array}$ & $\begin{array}{c}4.4816891 \\
(4.4816891)\end{array}$ \\
\hline 1 & $\begin{array}{c}1.2840254 \\
(1.2840254)\end{array}$ & $\begin{array}{c}1.5168968 \\
(1.5168968)\end{array}$ & $\begin{array}{c}1.7920018 \\
(1.7920018)\end{array}$ & $\begin{array}{c}2.1170000 \\
(2.1170000)\end{array}$ & $\begin{array}{c}2.5009400 \\
(2.5009400)\end{array}$ & $\begin{array}{c}2.9545115 \\
(2.9545115)\end{array}$ & $\begin{array}{c}3.4903430 \\
(3.4903430)\end{array}$ \\
\hline 0 & $\begin{array}{c}1.0000000 \\
(1.0000000)\end{array}$ & $\begin{array}{c}1.1813604 \\
(1.1813604)\end{array}$ & $\begin{array}{c}1.3956124 \\
(1.3956124)\end{array}$ & $\begin{array}{c}1.6487213 \\
(1.6487213)\end{array}$ & $\begin{array}{c}1.9477340 \\
(1.9477340)\end{array}$ & $\begin{array}{c}2.3009759 \\
(2.3009759)\end{array}$ & $\begin{array}{c}2.7182818 \\
(2.7182818)\end{array}$ \\
\hline
\end{tabular}

Table 5. The convergence of $\omega_{k}$.

\begin{tabular}{cccc}
\hline$\omega_{2}$ & $\omega_{3}$ & $\omega_{4}$ & $\omega_{5}$ \\
\hline 1.1269983 & 1.0086468 & 1.0000778 & 1.0000000 \\
\hline
\end{tabular}

method we need two first approximations $\omega_{0}$ and $\omega_{1}$ to $\omega$. In this example we were choose $\omega_{0}=3$ and $\omega_{1}=2$. The exact value of $\omega$ is $\omega=1$. The convergence of $\omega_{k}$ was tabulated in Table 5. The iteration was terminated by criterion (5.6) with $\varepsilon=10^{-7}$.

\section{REFERENCES}

[1] R. P. Agarwal, "Difference Equations and Inequalities: Theory, Methods and Applications," 2nd Edition, CRC
Press, Boca Raton, 2000.

[2] R. E. Mickens, "Nonstandard Finite Difference Models of Differential Equations," World Scientific, Singapore, 1994.

[3] A. A. Samarskii, "Theory of Difference Equations," 1977.

[4] B. Batgerel and T. Zhanlav, "An Exact Finite-Difference Scheme for Sturm-Liouville Problems," South Carolina, Vol. 1, No. 120, 1996, pp. 8-15. 